\title{
Chemical Hemisynthesis of Sulfated Cyanidin-3-O-Glucoside and Cyanidin Metabolites
}

\author{
Sarah Straßmann, Maike Passon *(D) and Andreas Schieber
}

Citation: Straßmann, S.; Passon, M.; Schieber, A. Chemical Hemisynthesis of Sulfated Cyanidin-3-O-Glucoside and Cyanidin Metabolites. Molecules 2021, 26, 2146. https://doi.org/ $10.3390 /$ molecules 26082146

Academic Editors: Toyonobu Usuki, Akira Kawamura and

Masaru Hashimoto

Received: 5 March 2021

Accepted: 6 April 2021

Published: 8 April 2021

Publisher's Note: MDPI stays neutral with regard to jurisdictional claims in published maps and institutional affiliations.

Copyright: (c) 2021 by the authors. Licensee MDPI, Basel, Switzerland. This article is an open access article distributed under the terms and conditions of the Creative Commons Attribution (CC BY) license (https:/ / creativecommons.org/licenses/by/ $4.0 /)$.
Department of Nutritional and Food Sciences, Molecular Food Technology, University of Bonn, Friedrich-Hirzebruch-Allee 7, D-53115 Bonn, Germany; sarah.strassmann@magenta.de (S.S.); schieber@uni-bonn.de (A.S.)

* Correspondence: passon@uni-bonn.de; Tel.: +49-228-73-4107
Abstract: The metabolism of anthocyanins in humans is still not fully understood, which is partly due to the lack of reference compounds. It is known that sulfation is one way of the complex phase II biotransformation mechanism. Therefore, cyanidin-3-O-glucoside and the cyanidin aglycone were chemically converted to their sulfates by reaction with sulfur trioxide-N-triethylamine complex in dimethylformamide. The reaction products were characterized by UHPLC coupled to linear ion trap and IMS-QTOF mass spectrometry. Based on MS data, retention times, and UV-Vis spectra, the compounds could tentatively be assigned to A-, C-, or B-ring sulfates. Analysis of urine samples from two volunteers after ingestion of commercial blackberry nectar demonstrated the presence of two sulfated derivatives of the cyanidin aglycone and one sulfated derivative of the cyanidin-3-Oglucoside. It was found that both the A ring and the B ring are sulfated by human enzymes. This study marks an important step toward a better understanding of anthocyanin metabolism.

Keywords: anthocyanins; cyanidin-3-O-glucoside; cyanidin; LC-IMS; HRMS; sulfation; metabolites

\section{Introduction}

Anthocyanins are water-soluble flavonoids that are widely found in the plant kingdom, for example, in berry fruits. Whereas the profile of some berries such as blueberries is rather complex, other fruits like strawberries and blackberries show a relatively simple composition, with pelargonidin-3-O-glucoside and cyanidin-3-O-glucoside respectively being the predominant compounds [1].

While it is well known that anthocyanins are poorly bioavailable, a large body of evidence suggests that they impart health benefits. Because anthocyanins have been demonstrated to be extensively metabolized, it is quite obvious that the metabolites rather than the parent compounds constitute the bioactive agents [2]. Most research on anthocyanin metabolites has focused on low-molecular phenolic compounds that result from the degradation of the flavonoid aglycone. However, metabolism of the intact anthocyanins and the aglycone may also occur, for example, by sulfation, glucuronidation, and methylation. In contrast to studies on the latter two pathways, the sulfation of anthocyanins has less frequently been reported, which may be due to the fact that sulfated metabolites are formed less often [3], the lack of reference compounds, and difficulties in sample preparation. However, the availability of standard substances is a prerequisite for the elucidation of the pharmacokinetics of anthocyanins.

Significant progress has been made in the synthesis of metabolites by enzymatic routes. For example, sulfated derivatives of cyanidin and cyanidin-3-O-glucoside were obtained employing porcine liver enzymes [4] and may be used for reference in databases. Because the yields were poor and enzymatic hemisynthesis is expensive, alternate approaches are needed.

Chemical hemisynthesis may be a more suitable tool to generate sulfated metabolites of anthocyanins. However, the sensitivity of anthocyanins to light, $\mathrm{pH}$ changes, and high 
temperature poses a problem [5], as does the lability of the sulfate groups under acidic conditions and at high temperatures [6]. As a result of these stability issues, synthesis should be carried out with as few steps as possible and under mild conditions. In anthocyanins, two types of hydroxyl groups are present, which are the aromatic $\mathrm{OH}$ groups of the anthocyanidin aglycone and the aliphatic $\mathrm{OH}$ groups of the sugar moiety. Because to our knowledge, there is no report about sulfations in the human body taking place on the sugar hydroxyl groups of anthocyanins, we aimed at the chemical hemisynthesis of anthocyanins sulfated on the flavonoid moiety. For this purpose, we used cyanidin-3-Oglucoside obtained from blackberry juice and the cyanidin aglycone prepared according to a previously described protocol [4]. Subsequently, we conducted a pilot study with two participants, who consumed blackberry juice and provided urine samples for analysis by LC-MS. This, in turn, was compared to the synthesized reference substances. Among other phase II metabolites of cyanidin, sulfated cyanidin-3-O-glucoside and sulfated cyanidin were detected. The aim of this work was to generate authentic reference substances for the identification of sulfated metabolites in real human biological samples.

\section{Results and Discussion}

The present study aimed at the chemical hemisynthesis of sulfated cyanidin-3-Oglucoside and cyanidin. For this purpose, the anthocyanin was purified from blackberry juice because blackberries have a relatively simple profile of pigments, cyanidin-3-Oglucoside being the predominant compound. Subsequently, the aglycone cyanidin was released by acidic hydrolysis. Both compounds reacted with a sulfur trioxide- $N$-triethylamine complex to yield a mixture of different mono- and disulfates (Scheme 1).

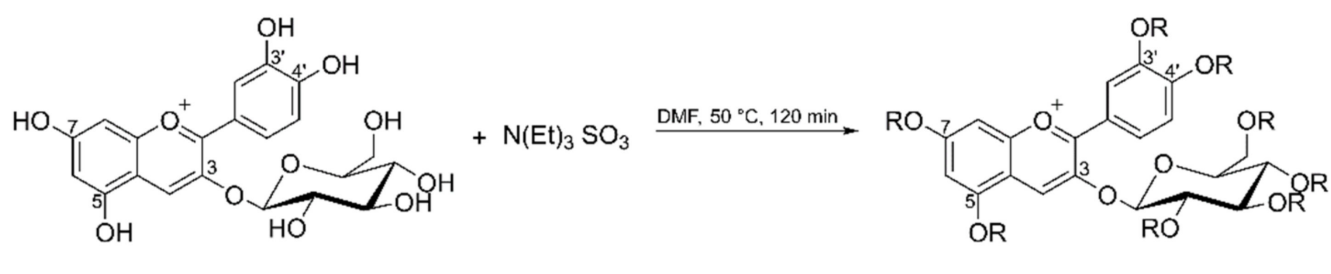<smiles>[R]Oc1ccc(-c2[o+]c3cc([R9])cc([R])c3cc2[R2])cc1O[R2]</smiles>

Scheme 1. Synthesis reactions of cyanidin sulfates and cyanidin-3-O-glucoside sulfates.

\subsection{Cyanidin Sulfates}

The extracted ion chromatogram of the $[\mathrm{M}]^{+}$of monosulfated cyanidin is shown in Figure 1A. Five peaks belonging to monosulfates (1-5) and at least two peaks (RT 4.5 and $11.3 \mathrm{~min}$ ) which are in-source fragments of disulfates can be observed. While absolute quantification was not possible due to the lack of references, the relative distribution of the monosulfates could be determined. Thus, compound 1 was formed at $4 \%$, compound 2 at $1 \%$, compound 3 at $3 \%$, compound 4 at $50 \%$, and compound 5 at $42 \%$. 

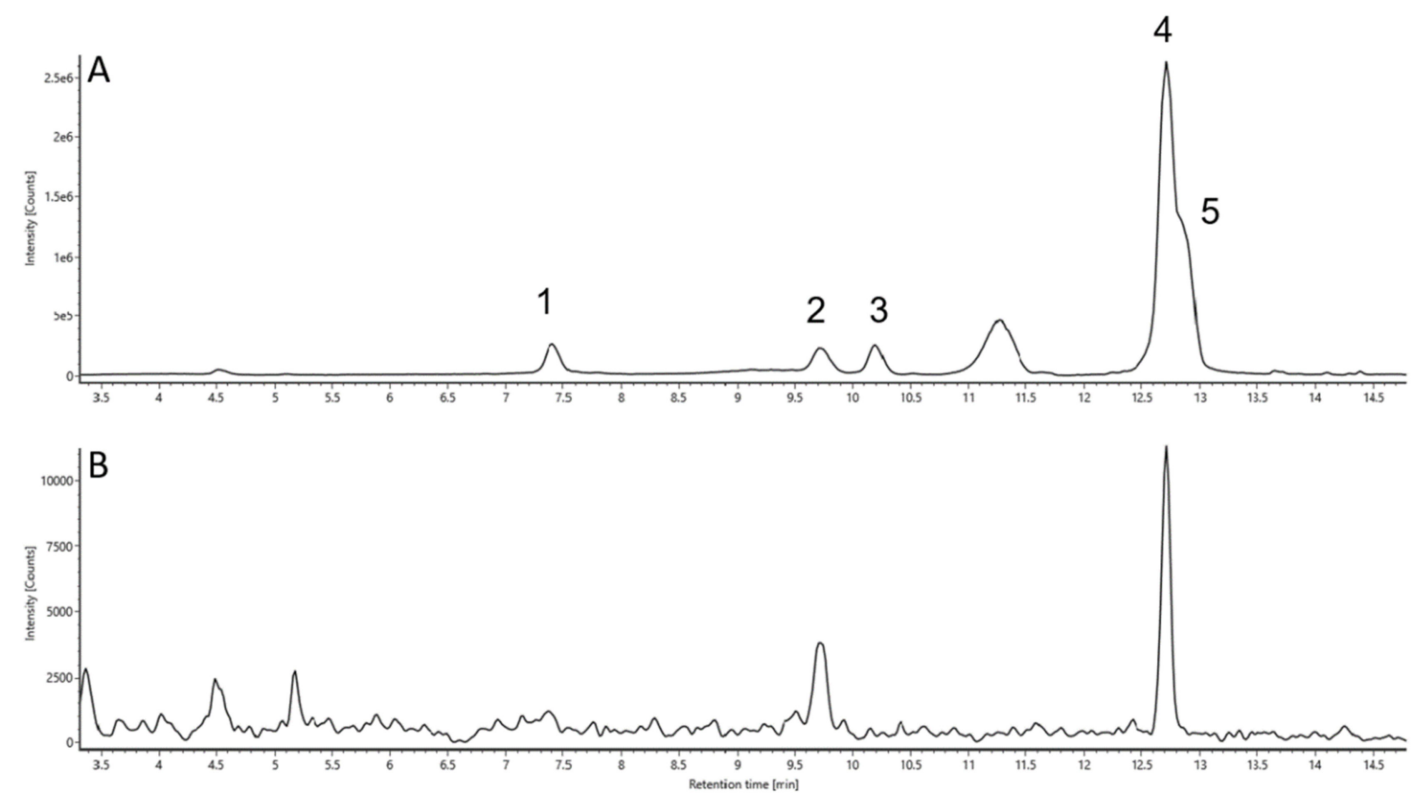

Figure 1. Extracted ion chromatograms of the $[\mathrm{M}]^{+}, m / z 367.0118$ (cyanidin monosulfate) after the synthesis reaction (A) and of the $24 \mathrm{~h}$ urine sample (B).

Identification was based on fragmentation patterns, accurate masses, CCS (collision cross-section) values, chromatographic behavior, and UV-spectra (Table 1). The interpretation of these data allows a tentative assignment of the site of sulfation to the A, B or C ring, which is described as follows.

\subsubsection{Retention Time}

The retention time may be indicative of the site of sulfation and should be taken into consideration for tentative peak assignment. From Figure 1A it can be seen that peak 1 elutes very early and remote from all other cyanidin sulfates. The chromatographic behavior of peaks 4 and $\mathbf{5}$ is very similar, resulting in partial coelution, which suggests a structural similarity. According to previous studies, quercetin sulfated at the $3-\mathrm{OH}$ position elutes earlier than derivatives that bear sulfate groups on the $3^{\prime}$ - or $4^{\prime}-\mathrm{OH}$ positions $[7,8]$. The elution order found for quercetin monosulfates was $3,5,7,4^{\prime}$, and $3^{\prime}$ [9]. Taking into account the structural similarity of flavonoids and the identical substitution pattern of the $\mathrm{B}$ ring of quercetin and cyanidin, it is reasonable to assume this elution order also for cyanidin monosulfaltes.

\subsubsection{UV/Vis Spectra}

Consideration of the shifts of the $\lambda_{\max }$ is another useful way of identifying the individual compounds formed in this reaction. Anthocyanins show a distinctive band I in the $450-560 \mathrm{~nm}$ region and band II in the $240-280 \mathrm{~nm}$ region [10,11]. Sulfation of cyanidin led to a hypsochromic shift in the spectrum of all peaks 1-5. Peak 1 showed the largest shift in comparison to cyanidin in band I from $525 \mathrm{~nm}$ to $505 \mathrm{~nm}$ and in band II from 277 to 267 $\mathrm{nm}$. Shifts in the range of 2 to $13 \mathrm{~nm}$ were observed for all other peaks. A comparison of the UV/Vis spectra of cyanidin, peak $\mathbf{1}$ and the two most abundant monosulfated cyanidin derivatives (peaks 4 and $\mathbf{5}$ ) is shown in Figure 2. Hypsochromic shifts of the absorption maxima of quercetin through sulfation are already reported in the literature [9]. Here, the $3-\mathrm{OH}$ sulfation caused also the highest shift of $21 \mathrm{~nm}$ in band I, whereas conjugation at the $5-, 4^{\prime}-$, and $3^{\prime}-\mathrm{OH}$ resulted in a small shift; sulfation of the 7-OH position did not affect the $\lambda_{\max }$. Interestingly, the formation of a new band at approximately $420 \mathrm{~nm}$ was observed, which constitutes an even bigger difference between the sulfated cyanidin molecules than the shift of the maxima. Peaks 1 and 4 show this band at $411 \mathrm{~nm}$ and $425 \mathrm{~nm}$ respectively, whereas sulfation at the other positions either does not affect this band, or the compound 
concentrations were too low. However, it is possible to determine the ratio of the absorption at $440 \mathrm{~nm}$ and the absorption maximum $\left(\lambda_{440 \mathrm{~nm}} / \lambda_{\max }\right)$. This ratio is $70 \%$ for peaks $1-3$, $50 \%$ for peak 4 and $30 \%$ for peak 5 , cyanidin, and cyanidin-3-O-glucoside. This ratio is already described in the literature [12], where it is reported that the ratio of an anthocyanin 3-O-glucoside is twice as high as that of a 5-O-glucoside. Larger shifts are caused by the disulfates, as can be seen from Table 1. Due to the high number of possible combinations and low substance concentrations, no conclusion concerning the conjugation positions can be drawn.

Table 1. LC-DAD-ESI-LIT and LC-DAD-ESI-IMS-QTOF results of the synthesis reaction of cyanidin sulfates.

\begin{tabular}{|c|c|c|c|c|c|c|c|c|c|c|}
\hline $\begin{array}{l}\text { Peak } \\
\text { No. }\end{array}$ & $\begin{array}{l}\mathrm{RT}^{\mathrm{a}} \\
(\mathrm{min})\end{array}$ & $\begin{array}{c}{[\mathrm{M}]^{+}} \\
\text {Calculated } \\
(\mathrm{m} / \mathrm{z})\end{array}$ & $\begin{array}{l}{[\mathrm{M}]^{+}} \\
\text {Mea- } \\
\text { sured } \\
(m / z)\end{array}$ & $\begin{array}{l}\text { Mass } \\
\text { Error } \\
(\mathrm{mDa})\end{array}$ & $\begin{array}{c}\text { Characteristic } \\
\text { Fragment } \\
\text { Ions MS }{ }^{2} \\
(m / z) \text { QTOF }\end{array}$ & $\begin{array}{l}\text { Characteristic } \\
\text { Fragment } \\
\text { Ions MS } \text { MS }^{2} \\
(\mathrm{~m} / \mathrm{z}) \text { LIT }\end{array}$ & $\begin{array}{l}\text { Degree of } \\
\text { Sulfation }\end{array}$ & $\begin{array}{l}\text { CCS Value } \\
/[\mathbf{M}]^{+}\left(\AA^{2}\right)\end{array}$ & $\begin{array}{c}\lambda_{\max } \\
(\mathrm{nm})\end{array}$ & $\begin{array}{l}\text { Supposed } \\
\text { Location of } \\
\text { Sulfate } \\
\text { Group }\end{array}$ \\
\hline- & 4.4 & 446.9686 & 446.9691 & +0.5 & $\begin{array}{l}286.0469 \\
367.0120\end{array}$ & 287,367 & 2 & $198.9 \pm 0.6$ & $\begin{array}{c}250,385 \\
505\end{array}$ & n.d. \\
\hline- & 4.8 & 446.9686 & 446.9684 & -0.2 & $\begin{array}{l}287.0550 \\
367.0117\end{array}$ & 287,367 & 2 & $196.3 \pm 0.1$ & n.d. & n.d. \\
\hline- & 5.5 & 446.9686 & 446.9692 & +0.6 & $\begin{array}{l}287.0552, \\
367.0120\end{array}$ & 287,367 & 2 & $204.5 \pm 0.6$ & 278,487 & n.d. \\
\hline- & 6.5 & 449.1078 & 449.1079 & +0.1 & $\begin{array}{l}287.0551 \\
121.0285, \\
137.0234\end{array}$ & 287 & * & $204.6 \pm 0.2$ & 278,516 & \\
\hline 1 & 7.5 & 367.0118 & 367.0118 & \pm 0.0 & $\begin{array}{l}149.0235, \\
177.0183, \\
256.9752, \\
286.0492, \\
287.0551\end{array}$ & $\begin{array}{l}137,177, \\
257,287\end{array}$ & 1 & $183.6 \pm 0.3$ & $\begin{array}{l}267,410 \\
505\end{array}$ & $\mathrm{C}$ ring \\
\hline - & 7.6 & 446.9686 & 446.9689 & +0.3 & $\begin{array}{l}287.0550 \\
367.0121\end{array}$ & 287,367 & 2 & $203.7 \pm 0.3$ & 281,505 & n.d. \\
\hline- & 9.3 & 446.9686 & 446.9686 & \pm 0.0 & $\begin{array}{l}286.0473, \\
367.0123 \\
109.0284, \\
121.0285 \\
137.0235 \\
139.0390 \\
149.0238,\end{array}$ & 287,367 & 2 & $202.7 \pm 0.1$ & $\begin{array}{c}254,372 \\
490\end{array}$ & n.d. \\
\hline 2 & 9.9 & 367.0118 & 367.0118 & \pm 0.0 & $\begin{array}{l}177.0185, \\
216.9805, \\
218.9785, \\
269.0447, \\
286.0475, \\
287.0551, \\
121.0286, \\
137.0236, \\
139.0392,\end{array}$ & $\begin{array}{c}177,217 \\
257,269,287\end{array}$ & 1 & $183.3 \pm 0.1$ & 275,513 & A ring \\
\hline 3 & 10.3 & 367.0118 & 367.0120 & +0.2 & $\begin{array}{l}149.0239, \\
177.0184, \\
219.0265, \\
256.9752, \\
287.0552,\end{array}$ & $\begin{array}{l}137,177 \\
257,287\end{array}$ & 1 & $187.6 \pm 0.1$ & 281,517 & A ring \\
\hline- & 11.3 & 446.9686 & 446.9687 & +0.1 & $\begin{array}{l}286.0470 \\
367.0120\end{array}$ & 287,367 & 2 & $189.6 \pm 0.2$ & $\begin{array}{c}278, \\
329 \mathrm{sh}, \\
428.497\end{array}$ & n.d. \\
\hline- & 11.7 & 287.0550 & 287.0552 & +0.2 & $\begin{array}{l}\text { 109.0286, } \\
121.0286, \\
137.0236, \\
149.0236, \\
269.0448\end{array}$ & $\begin{array}{l}137,149 \\
177,269\end{array}$ & cyanidin & $163.4 \pm 0.1$ & $\begin{array}{c}277, \\
443 \text { sh, } 525\end{array}$ & \\
\hline 4 & 12.6 & 367.0118 & 367.0118 & \pm 0.0 & $\begin{array}{l}216.9807 \\
268.0370 \\
286.0471 \\
268.0370\end{array}$ & $137,269,287$ & 1 & $182.6 \pm 0.1$ & $\begin{array}{c}271,429 \\
513\end{array}$ & $\mathrm{~B}$ ring \\
\hline 5 & 12.8 & 367.0118 & 367.0118 & \pm 0.0 & $\begin{array}{l}269.0445, \\
286.0472, \\
287.0553\end{array}$ & $137,269,287$ & 1 & $180.8 \pm 0.2$ & 283,512 & $\mathrm{~B}$ ring \\
\hline
\end{tabular}

${ }^{*}$ Cyanidin-3-O-glucoside; n.d. $=$ not determined; ${ }^{\mathrm{a}}=$ gradient $\mathrm{a}$. 


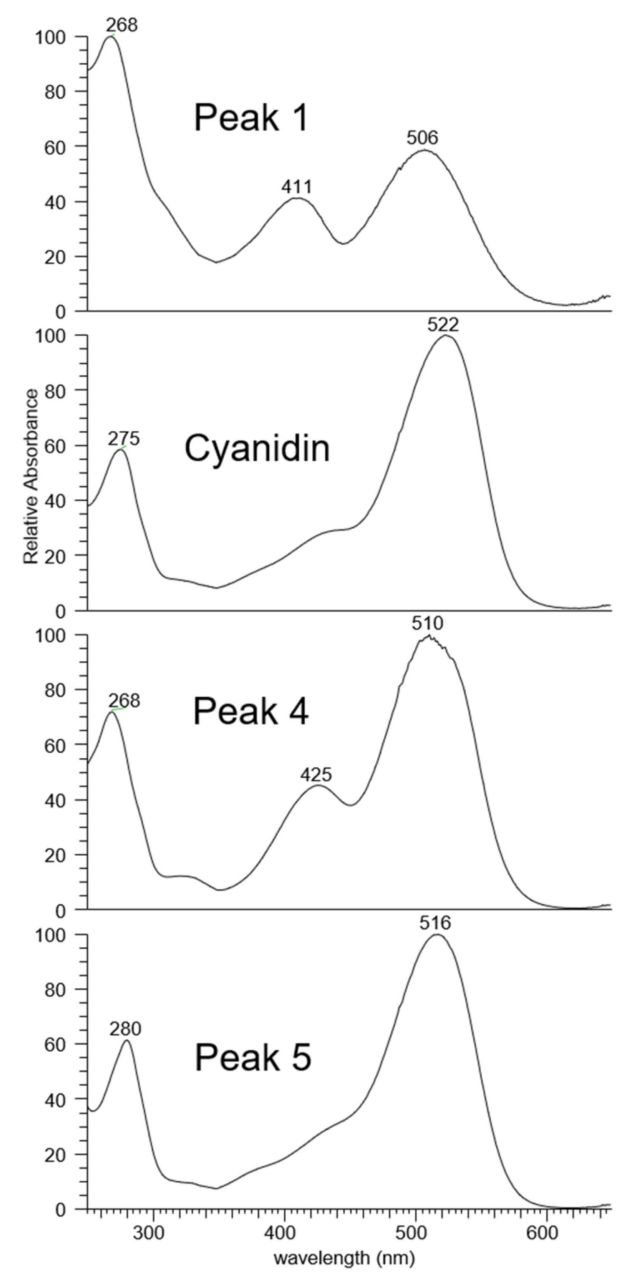

Figure 2. UV/Vis spectra of cyanidin (RT $11.7 \mathrm{~min}$ ) in comparison to three cyanidin monosulfates (RT 7.1, 12.6 and, $12.8 \mathrm{~min}$ ).

\subsubsection{Mass Spectrometry}

Mass spectrometry is one of the most valuable tools for the characterization of compounds and may even be superior to NMR spectroscopy when only small amounts are available. In the case of flavonoids, the small fragments emerging from the cleavage of the $\mathrm{C}$ ring are most valuable for identification purposes. The introduction of a sulfate group leads to a mass increment of $79.9569 \mathrm{u}$ in the spectrum. These characteristic fragments and their corresponding sulfated forms are shown in Figure 3 and the masses observed via LIT-MS or TOF-MS analyses are listed in Table 1 . Because the flavylium cation is very stable and the sulfate group represents a good leaving group leading to a neutral loss fragment, only limited fragmentation occurs and the fragments smaller than 286/287 u $\left([\mathrm{M}-80]^{+}\right)$ are of little diagnostic benefit. 


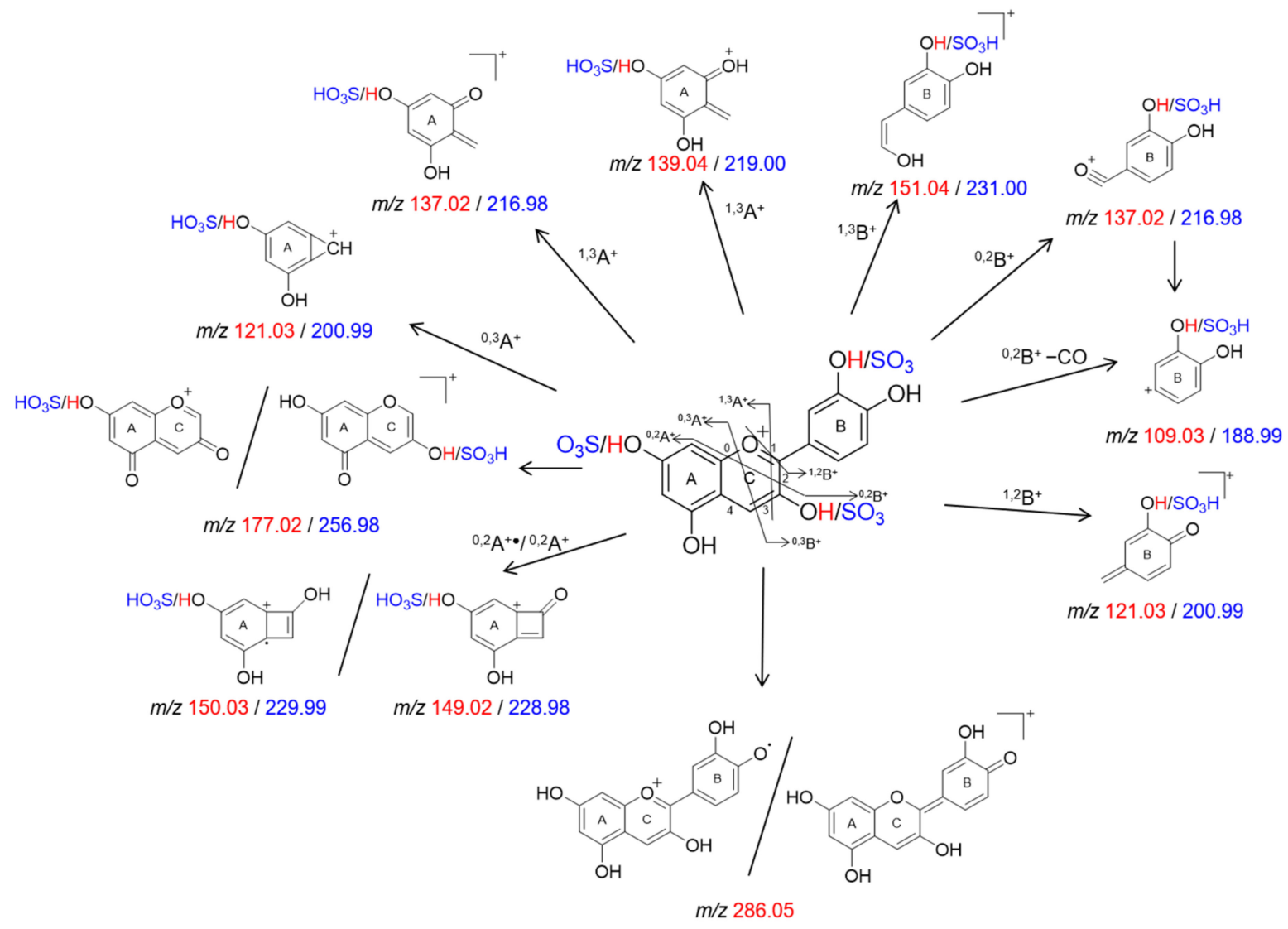

Figure 3. Postulated $\mathrm{ESI}^{+}$fragmentation scheme for cyanidin (red) and its derivatives (blue) sulfated on the A, B or C ring, based on the proposals of Barnes and Schug [13], González-Manzano et al. [14], and Oliveira et al. [15]. Conjugation positions are arbitrarily selected and may occur at any $\mathrm{OH}$ group.

However, a tentative assignment of the individual peaks to a conjugation position on the A-, B- or $\mathrm{C}$ ring is possible. Peak 1 shows numerous small fragments. The fragment at $m / z 149.02$ originates from the cleavage of the A ring $\left(0,2 \mathrm{~A}^{+}\right)$. Since it is not present in sulfated form, sulfation on the A ring can be excluded. Furthermore, fragments at $m / z$ $121.03\left({ }^{0,3} \mathrm{~A}^{+}\right.$or $\left.{ }^{1,2} \mathrm{~B}^{+}\right)$and $137.02\left({ }^{1,3} \mathrm{~A}^{+}\right.$or $\left.{ }^{0,2} \mathrm{~B}^{+}\right)$were obtained, which may be both $\mathrm{A}$ - and $\mathrm{B}$ ring fragments. Because no fragment corresponding to the sulfated form was observed, it can be concluded that sulfation took place on the $\mathrm{C}$ ring. This assumption is confirmed by the simultaneous presence of a fragment at $m / z 177.02\left(\left[\mathrm{M}-\mathrm{C}_{6} \mathrm{H}_{5} \mathrm{O}_{2}\right]^{+}\right)$and its sulfated form at $m / z 256.98$.

The spectra of peaks 2 and 3 look very similar, in here fragments at $m / z 137.02\left(1,3 \mathrm{~A}^{+}\right.$ or $\left.{ }^{0,2} \mathrm{~B}^{+}\right), 139.04\left({ }^{1,3} \mathrm{~A}^{+}\right)$, and the respective sulfated forms at $m / z 216.98$ and 219.00 are detectable. In addition, fragments at $m / z 177.02\left(\left[\mathrm{M}-\mathrm{C}_{6} \mathrm{H}_{5} \mathrm{O}_{2}\right]^{+}\right)$and 256.98 are present. Furthermore, the fragment at $m / z 109.03\left({ }^{0,2} \mathrm{~B}^{+}-\mathrm{CO}\right)$ but not its sulfated version can be found. These combinations lead to the conclusion that the A ring carries the sulfate group in these two compounds.

Peaks $\mathbf{4}$ and $\mathbf{5}$ do not show characteristic fragments, but also look very similar. However, applying the exclusion principle and considering their very similar retention times it can be concluded that these compounds correspond to cyanidin sulfated at the $3^{\prime}-\mathrm{OH}$ and $4^{\prime}-\mathrm{OH}$ groups of the B ring, respectively.

All peaks show a fragment at $m / z 286.05$, but with different intensities. Interestingly, the ratio of the fragment at $m / z 286.05$ to 287.02 is relatively constant and is 0.04 for peak $\mathbf{1}$, 0.07 for peak 2, and 0.01 for peak 3. In peak 4, this ratio reverses to 17 and the fragment at $\mathrm{m} / z 287.02$ is hardly detectable. The last peak (5) shows both fragments, in some analyses with a ratio of 0.07 and in some with a ratio of 17 . A possible explanation is that the fragment at $m / z 286.05$ belongs to one of the structures shown in Figure $3\left([\mathrm{M}-80]^{+}\right.$or 
$\left.[\mathrm{M}-80]^{+}\right)$and may be related to the radical scavenging properties of flavonoids [16]. The $o$-dihydroxy structure in the $\mathrm{B}$ ring confers high stability to a radical form and participates in electron delocalization [17].

It should be mentioned that some fragments were found only in the mass spectra recorded with the ion trap and some only in the spectra recorded with the TOF-MS. These differences may be due to the different collision gases used. The fragments are listed in Table 1 and therefore the differences are not explicitly mentioned in the descriptions of the spectra.

Another useful tool to distinguish compounds is ion mobility spectrometry and the resulting CCS values. If the measurements were taken on the same day, the values are very constant. In the case of the cyanidin sulfates, the CCS values are in the range of $180.8 \AA^{2}$ for a probable B ring sulfate and $187.6 \AA^{2}$ for a probable A ring sulfate. Thus, the distinction of the individual components can be accomplished based on these CCS values.

Founded on the data discussed above, the elution sequence of the monosulfated compounds is $\mathrm{C}$ ring sulfate $<\mathrm{A}$ ring sulfate $<\mathrm{B}$ ring sulfate.

\subsection{Cyanidin-3-O-Glucoside Sulfates}

In contrast to the cyanidin aglycone, there is no free $\mathrm{OH}$ group in the $\mathrm{C}$ ring that is available for sulfation. Therefore, conjugation of cyanidin-3-O-glucoside can take place only on the A- and the B rings. Figure 4 shows extracted ion chromatograms at $m / z 529.06$ and 609.02 , corresponding to the masses of mono- and disulfated cyanidin-3-O-glucoside.

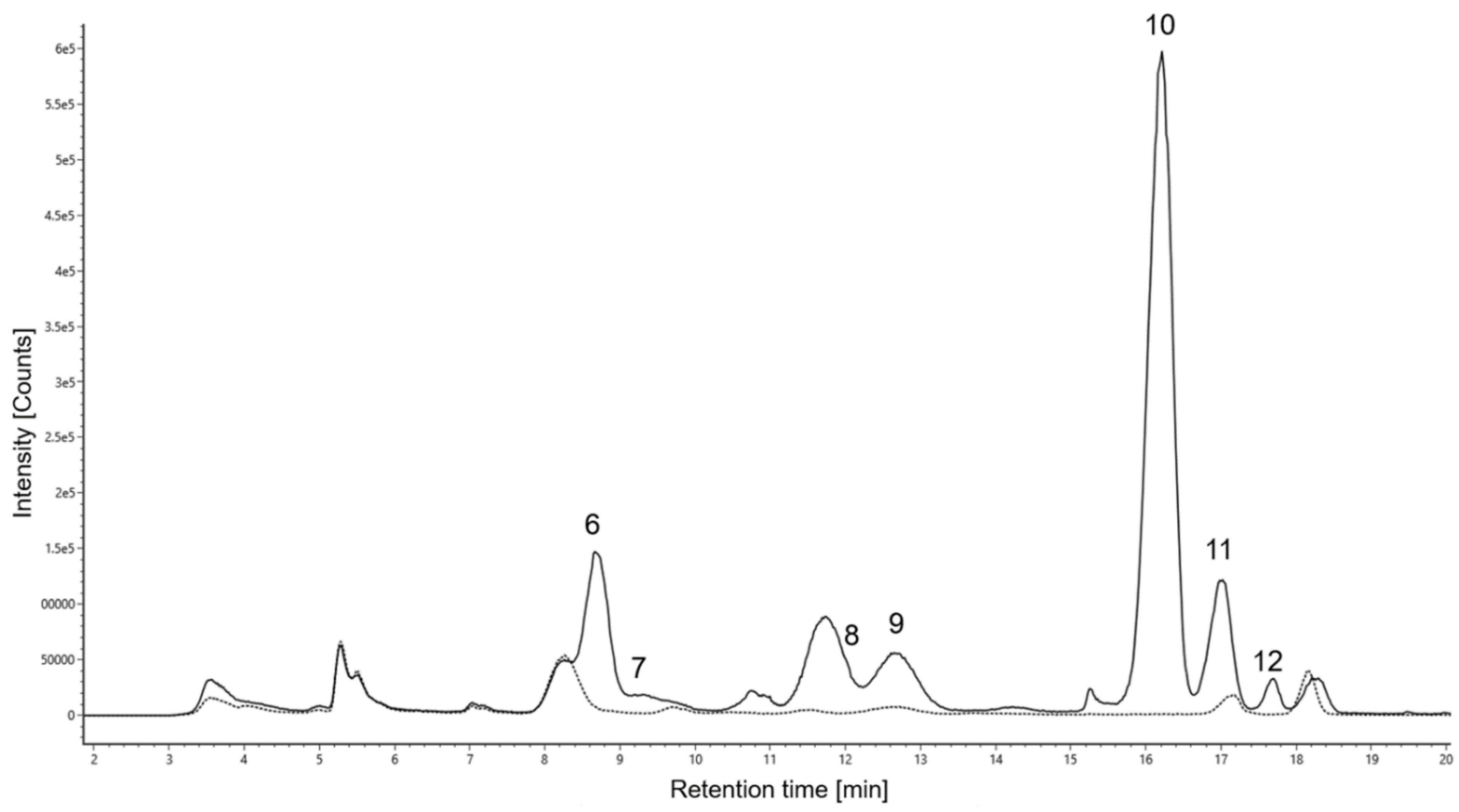

Figure 4. Extracted ion chromatograms of the $[\mathrm{M}]^{+}, m / z$ 529.0646 (black line) and 609.0215 (dashed line) (cyanidin-3-Oglucoside mono- and disulfate) after the synthesis reaction.

The yields determined at $280 \mathrm{~nm}$ were $4.4 \%$ for compound $6,3.0 \%$ for compound 8 , $3.8 \%$ for compound $\mathbf{9}$, and $26.9 \%$ for compound $\mathbf{1 0}$. All other monosulfated compounds were obtained at yields under $1 \%$. Table 2 illustrates the data used for identification purposes, that is, retention times, accurate masses, CCS values, $m / z$ of the most abundant fragments, relative yield, and absorption maxima. 
Table 2. LC-DAD-ESI-LIT and LC-DAD-ESI-IMS-QTOF results of the synthesis reaction of cyanidin-3-O-glucoside sulfates.

\begin{tabular}{|c|c|c|c|c|c|c|c|c|}
\hline Peak No. & $\begin{array}{l}\mathrm{RT}^{\mathrm{b}} \\
(\mathrm{min})\end{array}$ & $\begin{array}{c}{[\mathrm{M}]^{+}} \\
\text {Calculated } \\
(m / z)\end{array}$ & $\begin{array}{c}{[\mathrm{M}]^{+}} \\
\text {Measured } \\
(\mathrm{m} / \mathrm{z})\end{array}$ & $\begin{array}{c}\text { Mass Error } \\
\text { (mDa) }\end{array}$ & $\begin{array}{l}\text { Fragment Ions } \\
\operatorname{MS}^{2}(m / z)\end{array}$ & $\begin{array}{l}\text { Degree of } \\
\text { Sulfation }\end{array}$ & $\begin{array}{l}\text { CCS Value } \\
{[\mathrm{M}]^{+}\left(\AA^{2}\right)}\end{array}$ & $\lambda_{\max }$ \\
\hline- & 5.3 & 609.0215 & 609.0221 & +0.6 & $\begin{array}{c}529(3.5), 447 \\
(100), 367(4.2)\end{array}$ & 2 & $240.8 \pm 0.1$ & 277,505 \\
\hline- & 5.5 & 609.0215 & 609.0219 & +0.4 & $\begin{array}{c}529(5.1), 447 \\
(100), 367(5.4)\end{array}$ & 2 & $240.6 \pm 0.1$ & 278,505 \\
\hline- & 7.0 & 609.0215 & 609.0225 & +1.0 & $\begin{array}{c}529(100), 447 \\
(88.1), 367 \\
(52.3), 287(4.0)\end{array}$ & 2 & $237.5 \pm 0.5$ & 278 \\
\hline- & 8.3 & 609.0215 & 609.0224 & +0.9 & $\begin{array}{c}529(70.9), 367 \\
(100), 287(14.5)\end{array}$ & 2 & $232.3 \pm 0.1$ & 278,500 \\
\hline 6 & 8.8 & 529.0647 & 529.0650 & +0.3 & $\begin{array}{c}449(2.8), 367 \\
(100), 287(5.3)\end{array}$ & 1 & $224.7 \pm 0.1$ & 278,509 \\
\hline 7 & 9.3 & 529.0647 & 529.0649 & +0.2 & $\begin{array}{l}449(96.5), 367 \\
(3.0), 287(100)\end{array}$ & 1 & $217.1 \pm 0.1$ & 277,504 \\
\hline- & 9.8 & 609.0215 & 609.0223 & +0.8 & $\begin{array}{c}529(100), 447 \\
(2.3), 367(18.7) \\
287(3.3)\end{array}$ & 2 & $239.9 \pm 0.2$ & 278,509 \\
\hline- & 11.5 & 609.0215 & 609.0222 & +0.7 & $\begin{array}{c}529(57.4), 447 \\
(1.9), 367(100) \\
287(27.4)\end{array}$ & 2 & $236.2 \pm 0.1$ & 278,492 \\
\hline 8 & 12.0 & 529.0647 & 529.0652 & +0.5 & $\begin{array}{c}449(7.5), 367 \\
(100), 287(4.5)\end{array}$ & 1 & $219.2 \pm 0.1$ & 278,511 \\
\hline 9 & 12.8 & 529.0647 & 529.0650 & +0.3 & $\begin{array}{c}449(100), 367 \\
(80.2), 287 \\
(92.6)\end{array}$ & 1 & $216.2 \pm 2.4$ & $\begin{array}{c}277,407, \\
493\end{array}$ \\
\hline - & 15.2 & 449.1078 & 449.1079 & +0.1 & $287(100)$ & * & $204.6 \pm 0.2$ & 279,516 \\
\hline 10 & 16.4 & 529.0647 & 529.0651 & +0.4 & $\begin{array}{c}449(100), 287 \\
(99.6)\end{array}$ & 1 & $215.7 \pm 0.2$ & 279,516 \\
\hline 11 & 17.1 & 529.0647 & 529.0651 & +0.4 & $\begin{array}{c}449 \text { (100), } 287 \\
(35.2)\end{array}$ & 1 & $218.2 \pm 0.1$ & 280,516 \\
\hline- & 17.2 & 609.0215 & 609.0224 & +0.9 & $\begin{array}{c}529(100), 449 \\
(2.3), 367(1.8) \\
287(19.3)\end{array}$ & 2 & $224.7 \pm 0.2$ & 280,516 \\
\hline 12 & 17.7 & 529.0647 & 529.0648 & +0.1 & $\begin{array}{c}449 \text { (17.2), } 287 \\
(100)\end{array}$ & 1 & $214.9 \pm 0.1$ & 280,516 \\
\hline- & 18.2 & 609.0215 & 609.0220 & +0.5 & $\begin{array}{c}529(100), 449 \\
(21.8), 367(1.1) \\
287(3.9)\end{array}$ & 2 & $224.4 \pm 0.1$ & 280,517 \\
\hline
\end{tabular}

${ }^{*}$ cyanidin-3-O-glucoside; ${ }^{\mathrm{b}}=$ gradient $\mathrm{b}$.

\subsubsection{Retention Time}

Four compounds $(\mathbf{6}, \mathbf{7}, \mathbf{8}, \mathbf{9})$ at $m / z 529.06$ elute earlier and three $(\mathbf{1 0}, \mathbf{1 1}, \mathbf{1 2})$ later than cyanidin-3-O-glucoside. From this elution behavior it can be concluded that these two clusters differ in the site of conjugation. Furthermore, the first cluster can be divided into two groups. Peaks 6 and 7 elute at similar retention times as well as peaks $\mathbf{8}$ and $\mathbf{9}$. Considering the elution order of the sulfated cyanidin aglycone and assuming that the sugar moiety does not influence the elution behavior, the two earlier eluting peaks can be allocated to A ring sulfates and the other two to B ring sulfates.

\subsubsection{UV Spectra}

UV spectral data obtained for sulfation of cyanidin-3-O-glucoside were generally similar to those described for the aglycone. Because of the low concentrations, however, intensities were less pronounced. The absorptions at approximately $280 \mathrm{~nm}$ remained largely unchanged. Sulfation of compound 9 led to the largest shift of the $\lambda_{\max }$, that is, from $516 \mathrm{~nm}$ for cyanidin-3-O-glucoside to $493 \mathrm{~nm}$. The additional band at about $420 \mathrm{~nm}$ 
already described for the cyanidin sulfates (peak 1 and 4) was observed at $407 \mathrm{~nm}$. Based on this data, the above-mentioned peak assignment is confirmed.

\subsubsection{Mass Spectrometry}

The mass spectrometric data (Table 2) allowed the distinction of the above-mentioned two clusters of peaks. Compounds eluting earlier than 15 min showed a neutral loss of $162 \mathrm{u}$ caused by the loss of the glucose moiety. These carry the sulfate group at the aglycone. In contrast, for molecules eluting later than $15 \mathrm{~min}$, a neutral loss of $242 \mathrm{u}$ was observed, which is indicative of a sulfate group at the sugar moiety. Unfortunately, an unambiguous assignment is not possible to a distinct position by means of mass spectrometry because the sulfate group is an excellent neutral loss fragment. However, the intensities of the resulting fragment signals $\left.\left(\mathrm{m} / \mathrm{z} 287.06[\mathrm{M}-80-162]^{+}, 367.01[\mathrm{M}-162]^{+} \text {, and 449.11, [M-80] }\right]^{+}\right)$ differ for each peak (Table 2). This behavior can be exploited, in addition to the retention time, for the identification of metabolites in physiological samples when their isolation is not possible, and references are not available. Because the four peaks eluting early (compounds 6, 7, 8 and 9) could be allocated to a sulfated aglycone, it is evident that all hydroxyl groups are sulfated, yet some of them seem to be preferred. Whereas compound 6 was obtained at a yield of $4.5 \%$, compound 7 was produced to such a small extent that no yield could be determined. This preference might be attributed to the differences in the $\mathrm{pK}_{\mathrm{a}}$ value of the hydroxyl groups, which is the lowest for the 5-OH group [18]. In the case of the sugar moiety, steric reasons may be the explanation for differences in the site of sulfation [19]. Furthermore, higher temperatures during the reaction lead to an increased formation of disulfates, which is described in the literature [19] and which was also observed in this work.

Interestingly, we observed decreasing CCS values with increasing retention times within the monosulfates or disulfates, respectively. It may be concluded that smaller molecules, whose size can be derived from the shorter drift times, interact more with the column material, which results in longer retention times. This observation was also made with the metabolites that resulted from cyanidin and cyanidin-3-O-glucoside through liver enzymes [4].

\subsection{Challenges}

Some difficulties with the sulfation of small molecules are already described in the literature [6]. As inorganic salts may pose a problem, particularly in smaller approaches, they may cause inconsistencies concerning the purification and thus the yield. While the separation of quercetin sulfates by preparative HPLC has already been reported to pose a problem, [20] it may be even more difficult in the case of anthocyanins because of their $\mathrm{pH}$-dependent structure. Additionally, the instability of the sulfate groups under acidic conditions and at high temperatures are pointed out in the literature [6]. The synthesis of resveratrol sulfates has even been referred to as a nightmare that can be handled only by selective protection of the $\mathrm{OH}$ groups [21]. Protective groups, which are often used for flavonoid derivatization, should be avoided for anthocyanins because their introduction and removal are often associated with unfavorable conditions. In contrast, the method applied in this study allows the production of sulfated derivatives without using protective groups. Another issue is the solvolysis of phenolic sulfates, which has been reported for 3 - and 4-O-resveratrol sulfates during evaporation to dryness, probably caused by the presence of sulfuric acid as a reaction byproduct [22]. These and other challenges are the reasons why anthocyanins rarely appear in the long history of sulfation of phenolic substances [23]. Furthermore, with respect to the intensities, it should also be considered that the anthocyanin monosulfates may be converted to disulfates. Therefore, fewer monosulfates should be present at higher temperatures and longer reaction times.

Although LC-MS analysis is extremely useful for metabolite characterization, challenges still exist in the assignment of the peaks to the right number of sulfates. The retention 
times of some of the monosulfates are close to the disulfates and the disulfates produce monosulfates as in source fragments.

\subsection{Human Pilot Study}

In a first pilot study comprising only two individuals, we investigated urine samples for the presence of anthocyanin phase II metabolites after ingestion of a commercial blackberry nectar sample. The qualitative profile of the investigated metabolites in the two urine samples was comparable. Their analytical data such as the accurate mass, the main fragment, and the CCS values are given in Table 3. Among the metabolites detected, two sulfated cyanidin derivatives and one sulfated cyanidin-3-O-glucoside were found. The latter elutes after $13.1 \mathrm{~min}$ (gradient $\mathrm{b}$ ), which corresponds to the retention time of the reference sulfate 9 . Their CCS values are also in the same range. However, it should be noted that while the deviations observed within a measurement are remarkably small, larger differences between different measurements may occur. For example, day-to-day variations in the CCS value for cyanidin of $162.5 \pm 0.1 \AA^{2}$ and on another day $165.5 \pm 0.1 \AA^{2}$ were observed, which results in an overall deviation of $\pm 2.1 \AA^{2}$. Thus, the deviation of the CCS value of the synthesized sulfate $9\left(216.2 \pm 2.4 \AA^{2}\right)$ from the sulfate found in urine $\left(209.4 \pm 0.6 \AA^{2}\right)$ with nearly the same retention time can be justified.

Table 3. LC-DAD-ESI-IMS-QTOF results of the analysis of urine collected for $24 \mathrm{~h}$ after the consumption of blackberry juice, either gradient $a$ or $b$.

\begin{tabular}{|c|c|c|c|c|c|c|}
\hline $\begin{array}{l}\text { Suspected } \\
\text { Compound }\end{array}$ & RT (min) & $\begin{array}{c}{[\mathrm{M}]^{+} \text {Calculated }} \\
(\mathrm{m} / \mathrm{z})\end{array}$ & $\begin{array}{c}{[\mathrm{M}]^{+} \text {Measured }} \\
(\mathrm{m} / \mathrm{z})\end{array}$ & $\begin{array}{c}\text { Mass Error } \\
\text { (mDa) }\end{array}$ & $\begin{array}{c}\text { Fragment } \text { MS }^{2} \\
(m / z)\end{array}$ & $\begin{array}{c}\text { CCS Value } \\
\left(\AA^{2}\right)\end{array}$ \\
\hline \multicolumn{7}{|c|}{ Cyanidin } \\
\hline unconjugated * & $11.7^{\mathrm{a}}$ & 287.0550 & 287.0549 & -0.1 & & $162.5 \pm 0.1$ \\
\hline glucuronidated & $8.3^{\mathrm{a}}$ & 463.0877 & 463.0871 & -0.6 & 287 & $200.1 \pm 0.9$ \\
\hline & $9.6^{\mathrm{a}}$ & 463.0877 & 463.0874 & -0.3 & 287 & $204.7 \pm 1.0$ \\
\hline \multirow{2}{*}{$\begin{array}{l}\text { glucuronidated } \\
\text { and methylated }\end{array}$} & $8.8^{a}$ & 477.1033 & 477.1029 & -0.4 & 301 & $209.4 \pm 2.4$ \\
\hline & $9.1^{\mathrm{a}}$ & 477.1033 & 477.1030 & -0.3 & 301 & $207.6 \pm 0.7$ \\
\hline \multirow{4}{*}{ sulfated } & $9.6^{\mathrm{a}}$ & 477.1033 & 477.1028 & -0.5 & 301 & $208.2 \pm 0.7$ \\
\hline & $10.2^{\mathrm{a}}$ & 477.1033 & 477.1029 & -0.4 & 301 & $207.6 \pm 0.4$ \\
\hline & $9.8^{\mathrm{a}}$ & 367.0118 & 367.0118 & \pm 0.0 & 287 & $180.0 \pm 1.0$ \\
\hline & $12.7^{\mathrm{a}}$ & 367.0118 & 367.0118 & \pm 0.0 & 287 & $180.6 \pm 0.5$ \\
\hline \multicolumn{7}{|c|}{ Cyanidin-3-O-glucoside } \\
\hline unconjugated * & $15.0^{\mathrm{b}}$ & 449.1078 & 449.1081 & +0.3 & 287 & $203.3 \pm 2.5$ \\
\hline sulfated & $13.1^{b}$ & 529.0647 & 529.0648 & +0.1 & 287 & $209.4 \pm 0.6$ \\
\hline
\end{tabular}

$\mathrm{a}=$ gradient $\mathrm{a} ;{ }^{\mathrm{b}}=$ gradient $\mathrm{b} ;{ }^{*}$ Not found in the samples, listed only for comparison.

The earliest peak corresponding to cyanidin sulfates that were detected in the urine samples elutes after $9.8 \mathrm{~min}$ (gradient a), which matches the retention time of peak 2 . The CCS value of this compound is $180.0 \pm 1.0 \AA^{2}$ and hence approximately $2 \AA^{2}$ below that of the reference compound, which can be explained by the fact that measurements were not taken on the same day.

Taking into account these deviations, the compound eluting after $12.7 \mathrm{~min}$ can be assigned to peak 4 of the synthesized sulfates. The CCS value also of this peak 4 is approximately $2 \AA^{2}$ higher than that of the substance detected in the urine.

Based on the mass spectrometric data, the compound eluting at $9.8 \mathrm{~min}$ can be assigned to an A ring sulfate, whereas the compound eluting after $12.7 \mathrm{~min}$ is sulfated at the B ring. To the best of our knowledge, this is the first work that reports a flavonoid metabolized to an A ring sulfate. Studies conducted on quercetin demonstrated the formation of quercetin$3^{\prime}$-O-sulfate in rat liver [7], which is a metabolite also present in humans [8]. From these findings, it may be deduced that compound 4 corresponds to cyanidin-3'-O-sulfate. As the $3^{\prime}$-O-sulfate was also obtained in the highest yields by other chemical syntheses [7], it may be concluded that the most intense peak in the synthesis of cyanidin sulfates is the $3^{\prime}-O-$ 
sulfate. These results would confirm that the human body produces mainly $3^{\prime}$-O-sulfates of flavonoids [8].

\section{Materials and Methods}

\subsection{Chemicals}

LC-MS-grade water, acetonitrile, formic acid, and ammonium hydroxide were purchased from ChemSolute (Renningen, Germany). Sulfur trioxide- $N$-triethylamine complex, leucine enkephalin acetate salt hydrate, and pyridine were obtained from Sigma-Aldrich (St. Louis, MO, USA). N,N-Dimethylformamide (DMF) was from Fisher Scientific (Waltham, MA, USA). Methanol, ascorbic acid, and hydrochloric acid ( $\mathrm{HCl})$ were supplied by VWR (Darmstadt, Germany). Trifluoroacetic acid (TFA) was purchased from Alfa Aesar (Ward Hill, MA, USA). An anthocyanin extract containing primarily cyanidin-3-O-glucoside (Scheme 2) was obtained as described before [4]. To obtain the cyanidin aglycone, the lyophilized extract was treated with concentrated $\mathrm{HCl}$ for 90 min at $90^{\circ} \mathrm{C}$.

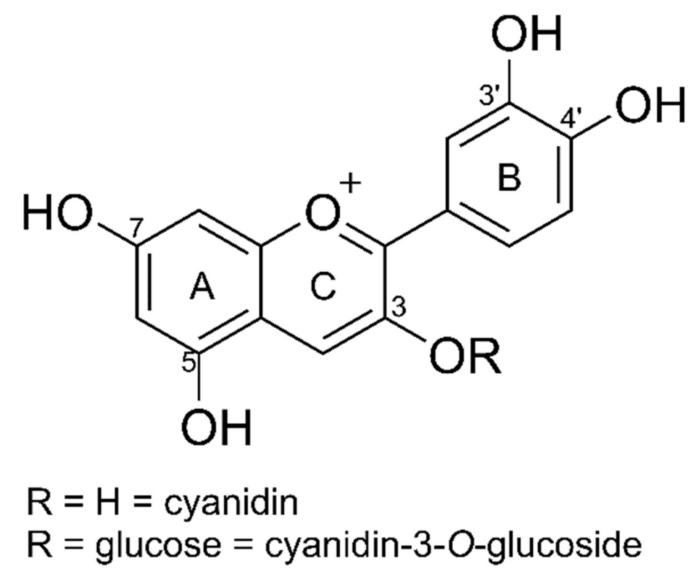

Scheme 2. Structure of cyanidin and cyanidin-3-O-glucoside.

\subsection{Hemisynthesis of Sulfated Metabolites}

The approach is based on the method described by Jones et al. [7] and the equations in Scheme 1 graphically illustrate the two reactions of cyanidin and cyanidin-3-O-glucoside, respectively. Cyanidin-3-O-glucoside $(7 \mu \mathrm{mol})$ was dissolved in approximately $20 \mathrm{~mL}$ dry pyridine to remove any water that may be present. Pyridine was evaporated under reduced pressure and the entire procedure was repeated. The dried compound was dissolved in $1 \mathrm{~mL}$ DMF. The cyanidin aglycone $(7 \mu \mathrm{mol})$ was dissolved directly in $1 \mathrm{~mL}$ DMF. This mixture was then transferred to an Eppendorf tube and allowed to react with a 10-fold excess of sulfur trioxide- $N$-triethylamine complex in a thermomixer (Eppendorf, Hamburg, Germany). A temperature of $50{ }^{\circ} \mathrm{C}$ and a reaction time of $120 \mathrm{~min}$ were found to be optimal for the hemisynthesis of monosulfated cyanidin-3-O-glucoside; for the cyanidin aglycone, optimum conditions were $45^{\circ} \mathrm{C}$ and $60 \mathrm{~min}$, respectively. These conditions allowed both maximizing the concentration of the monosulfates and minimizing the formation of multiple sulfated conjugates. After 5-fold dilution with water and acetonitrile containing $0.1 \%$ formic acid, and microfiltration (regenerated cellulose, $0.20 \mu \mathrm{m}$ ), the samples were analysed using LC-LIT-MS ${ }^{\mathrm{n}}$ and LC-IMS-QTOF-MS.

The polarity of non-charged flavonoids is increased by sulfation in a way that they precipitate in the used solvent, which is mostly dioxane [7]. In the case of anthocyanins, the polarity of the resulting substances is similar to the parent molecules, so that they do not precipitate. Therefore, solid-phase extraction with a weak anion exchanger was applied to separate the cyanidin-3-O-glucoside sulfates from the starting material to take advantage of the different local charge distribution. For this purpose, OASIS WAX cartridges $(6 \mathrm{cc}$ Vac cartridge, $500 \mathrm{mg}$ sorbent per cartridge, $60 \mu \mathrm{m}$ particle size; Waters, Milford, MA, USA) were washed with methanol and water. After dilution of the samples with $4 \mathrm{~mL}$ 
water, aliquots of $2 \mathrm{~mL}$ were applied to the ion exchanger; subsequently, the material was washed again with water containing $2 \%$ of formic acid. Most of the unreacted cyanidin-3O-glucoside was removed using $100 \%$ methanol in the first elution step. The sulfated target compounds were then eluted with $5 \%$ ammonia in methanol, $3 \% \mathrm{HCl}$ in water/methanol 50:50 $(v / v)$, and 20\% trifluoracetic acid in water/methanol 50:50 $(v / v)$ until the eluates were colorless (approx. 3-5 mL). Eluates were evaporated under a constant nitrogen stream to dryness and redissolved in $250 \mu \mathrm{L}$ of acetonitrile/water/formic acid 20:79.9:0.1 $(v / v / v)$.

\subsection{LC-MS-Analysis}

UHPLC analysis of the reaction products was performed on an Acquity UPLC IClass system (Waters, Milford, MA, USA) consisting of a binary pump, a sample manager cooled at $10^{\circ} \mathrm{C}$, a column oven set at $40^{\circ} \mathrm{C}$, and a diode array detector scanning from 250 to $650 \mathrm{~nm}$. An Acquity HSS-T3 RP18 column $(150 \mathrm{~mm} \times 2.1 \mathrm{~mm} ; 1.8 \mu \mathrm{m}$ particle size $)$ combined with a pre-column (Acquity UPLC HSS T3 VanGuard, $100 \AA$, $2.1 \mathrm{~mm} \times 5 \mathrm{~mm}$, $1.8 \mu \mathrm{m}$ ), both from Waters (Milford, MA, USA) was used for separation with water (A) and acetonitrile $(\mathrm{B})$ as eluents, both acidified with $0.1 \%(\mathrm{v}+\mathrm{v})$ formic acid. The flow rate was set at $0.4 \mathrm{~mL} / \mathrm{min}$. Since the reaction products differ considerably in their polarity, two different gradients had to be applied to achieve the best separation possible. Sulfated reaction products of the cyanidin aglycone were analyzed using a linear gradient from $8 \%$ $\mathrm{B}$ to $10 \% \mathrm{~B}$ for $5 \mathrm{~min}$, then to $17 \% \mathrm{~B}$ for $6 \mathrm{~min}$, to $30 \% \mathrm{~B}$ for $4 \mathrm{~min}$, and to $50 \% \mathrm{~B}$ for $8 \mathrm{~min}$ (gradient a). Sulfates of cyanidin-3-O-glucoside were analyzed using a gradient whose solvent composition changed from 1 to $10 \% \mathrm{~B}$ in $13 \mathrm{~min}$, then to $17 \% \mathrm{~B}$ in $6 \mathrm{~min}$, and then to $30 \% \mathrm{~B}$ in $4 \mathrm{~min}$ (gradient $\mathrm{b}$ ). The injection volume was $5 \mu \mathrm{L}$. For MS analysis, the UHPLC was coupled with an LTQ-XL ion trap mass spectrometer (Thermo Scientific, Inc., Waltham, MA, USA) equipped with an electrospray interface operating in the positive ion mode. Ion mass spectra were recorded in the range of $m / z 245-1000$. The capillary was set at $325^{\circ} \mathrm{C}$ with a spray voltage of $16 \mathrm{~V}$ for cyanidin and $30 \mathrm{~V}$ for cyanidin-3-O-glucoside. The source voltage was maintained at $4 \mathrm{kV}$ at a current of $100 \mu \mathrm{A}$ and the tube lens was adjusted to $55 \mathrm{~V}$ for cyanidin and $75 \mathrm{~V}$ for cyanidin-3-O-glucoside. Nitrogen was used as sheath, auxiliary and sweep gas at a flow of 70,10 and $1 \mathrm{arb}$, respectively. Three consecutive scans were conducted with helium as collision gas: a full mass scan, an MS ${ }^{2}$ scan of the most abundant ion of the first scan using normalized collision energy (CE) of $20 \%$, and an $\mathrm{MS}^{3}$ analysis of the most abundant ion in the $\mathrm{MS}^{2}$ experiment with a CE of $35 \%$. In addition, multiple reaction monitoring (MRM) measurements were performed and the masses resulting from typical losses of one $[\mathrm{M}-80]^{+}$or two $[\mathrm{M}-80-80]^{+}$sulfate groups or glucose $[\mathrm{M}-162]^{+}$were scanned. Data were acquired and processed using Xcalibur 2.2SP1.48 (Thermo Scientific, Inc., Waltham, MA, USA).

For ion mobility spectrometry measurements, the UHPLC was connected to a Vion IMS QTOF mass spectrometer (Waters, Milford, MA, USA) operating in the positive ion mode. The capillary voltage was $0.5 \mathrm{kV}$, the source temperature was $120^{\circ} \mathrm{C}$, the cone voltage was $40 \mathrm{~V}$, the desolvation gas temperature was $550^{\circ} \mathrm{C}$, and the desolvation gas flow was $1200 \mathrm{~L} / \mathrm{h}$. The measurements were conducted with automatic lock correction every 5 min with leucine enkephalin as the lock mass at a concentration of $100 \mathrm{pg} / \mu \mathrm{L}$. Nitrogen was used as the drift and collision gas and the MS mode was high definition with a low collision energy of $6 \mathrm{eV}$ and a high collision energy ramp of 20-40 eV. Data were acquired and processed using UNIFI v1.9.2.045 (Waters, Milford, MA, USA).

\subsection{Study Design and Analysis of Urine}

As a proof of concept, the urine of two people was collected over $24 \mathrm{~h}$ after consumption of $400 \mathrm{~mL}$ commercial blackberry nectar. This volume contained about $100 \mathrm{mg}$ cyanidin-3-O-glucoside. Additionally, one sample of urine was taken prior to consumption.

The diet was anthocyanin-free in the week before the study and polyphenol-free on the day of the study. According to the procedure of Feliciano et al. [24], ascorbic acid was added to the urine containers (5.63 g/3 L container). Aliquots were adjusted to $\mathrm{pH} 2.5$ with 
formic acid for stabilization and measured directly to avoid precipitation of the desired metabolites [25]. Direct measurement was required because we had observed that after one month of storage of the samples at $-80^{\circ} \mathrm{C}$, the peak intensities were significantly lower.

\subsection{Sample Preparation}

Samples were prepared according to a previously published protocol [26]. Briefly, $5 \mathrm{~mL}$ of a urine sample was evaporated at $40{ }^{\circ} \mathrm{C}$ under nitrogen flow to a final volume of 500-700 $\mu \mathrm{L}$. After microfiltration (regenerated cellulose, $0.20 \mu \mathrm{m}$ ), the samples were analysed using LC-IMS-QTOF-MS and LC-LIT-MS.

\section{Conclusions}

Our knowledge about the fate of anthocyanins in the human body is still incomplete. It is known that after absorption various enzymes transfer glucuronic acid and/or a sulfate group as well as a methyl group to the aglycone or glycoside. Additionally, cleavage of the glycoside and other reactions are possible. From the results of the human pilot study, it is evident that the metabolite species mentioned above can be found in various forms in urine. It has been possible to chemically synthesize sulfates of cyanidin and cyanidin-3-Oglucoside and to use these as reference substances. Thus, two different cyanidin sulfates and one cyanidin-3-O-glucoside sulfate could be identified. In addition, two glucuronides and four combinations of glucuronidated and methylated derivatives of cyanidin were detected in human urine. Despite the difficulties associated with the small quantities from chemical synthesis, the structural elucidation of the sulfated anthocyanins is an advance in the complete characterization of their metabolic profile.

Author Contributions: Conceptualization, S.S., M.P. and A.S.; methodology, S.S.; validation, S.S.; investigation, S.S.; interpretation, S.S., M.P. and A.S.; resources, A.S.; writing-original draft preparation, S.S.; writing-review and editing, M.P. and A.S.; supervision, M.P. and A.S.; project administration, M.P. All authors have read and agreed to the published version of the manuscript.

Funding: This research received no external funding.

Institutional Review Board Statement: The study was conducted according to the guidelines of the Declaration of Helsinki, and approved by the ethics commission of the Medical Faculty of the University of Bonn (project identification code 019/17).

Informed Consent Statement: Informed consent was obtained from all subjects involved in the study.

Data Availability Statement: Data is contained within the article.

Conflicts of Interest: The authors declare no conflict of interest.

Sample Availability: Samples of the compounds are not available from the authors.

\section{References}

1. Horbowicz, M.; Kosson, R.; Grzesiuk, A.; Dębski, H. Anthocyanins of Fruits and Vegetables-Their Occurrence, Analysis and Role in Human Nutrition. Veg. Crop. Res. Bull. 2008, 68, 5-22. [CrossRef]

2. Kay, C.D.; Pereira-Caro, G.; Ludwig, I.A.; Clifford, M.N.; Crozier, A. Anthocyanins and Flavanones Are More Bioavailable than Previously Perceived: A Review of Recent Evidence. Annu. Rev. Food Sci. Technol. 2017, 8, 155-180. [CrossRef] [PubMed]

3. Felgines, C.; Talavera, S.; Texier, O.; Gil-Izquierdo, A.; Lamaison, J.-L.; Remesy, C. Blackberry Anthocyanins Are Mainly Recovered from Urine as Methylated and Glucuronidated Conjugates in Humans. J. Agric. Food Chem. 2005, 53, 7721-7727. [CrossRef] [PubMed]

4. Schmitt, S.; Tratzka, S.; Schieber, A.; Passon, M. Hemisynthesis of Anthocyanin Phase II Metabolites by Porcine Liver Enzymes. J. Agric. Food Chem. 2019, 67, 6177-6189. [CrossRef]

5. Fossen, T.; Cabrita, L.; Andersen, O.M. Colour and stability of pure anthocyanins influenced by pH including the alkaline region. Food Chem. 1998, 63, 435-440. [CrossRef]

6. Al-Horani, R.A.; Desai, U.R. Chemical sulfation of small molecules-Advances and challenges. Tetrahedron 2010, 66, 2907-2918. [CrossRef]

7. Jones, D.J.; Jukes-Jones, R.; Verschoyle, R.D.; Farmer, P.B.; Gescher, A. A synthetic approach to the generation of quercetin sulfates and the detection of quercetin $3^{\prime}-\mathrm{O}$-sulfate as a urinary metabolite in the rat. Bioorg. Med. Chem. 2005, 13, 6727-6731. [CrossRef] 
8. Day, A.J.; Mellon, F.; Barron, D.; Sarrazin, G.; Morgan, M.R.; Williamson, G. Human metabolism of dietary flavonoids: Identification of plasma metabolites of quercetin. Free Radic. Res. 2001, 35, 941-952. [CrossRef]

9. Dueñas, M.; González-Manzano, S.; Surco-Laos, F.; González-Paramas, A.; Santos-Buelga, C. Characterization of Sulfated Quercetin and Epicatechin Metabolites. J. Agric. Food Chem. 2012, 60, 3592-3598. [CrossRef]

10. Anouar, E.H.; Gierschner, J.; Duroux, J.-L.; Trouillas, P. UV/Visible spectra of natural polyphenols: A time-dependent density functional theory study. Food Chem. 2012, 131, 79-89. [CrossRef]

11. Welch, C.R.; Wu, Q.; Simon, J. Recent Advances in Anthocyanin Analysis and Characterization. Curr. Anal. Chem. 2008, 4, 75-101. [CrossRef]

12. Giusti, M.; Wrolstad, R.E. Characterization and Measurement of Anthocyanins by UV-Visible Spectroscopy. Curr. Protoc. Food Anal. Chem. 2001, 00, F1.2.1-F1.2.13. [CrossRef]

13. Barnes, J.S.; Schug, K.A. Structural characterization of cyanidin-3,5-diglucoside and pelargonidin-3,5-diglucoside anthocyanins: Multi-dimensional fragmentation pathways using high performance liquid chromatography-electrospray ionization-ion trap-time of flight mass spectrometry. Int. J. Mass Spectrom. 2011, 308, 71-80. [CrossRef]

14. González-Manzano, S.; González-Paramás, A.; Santos-Buelga, C.; Dueñas, M. Preparation and Characterization of Catechin Sulfates, Glucuronides, and Methylethers with Metabolic Interest. J. Agric. Food Chem. 2009, 57, 1231-1238. [CrossRef]

15. Oliveira, M.C.; Esperança, P.; Ferreira, M.A.A. Characterisation of anthocyanidins by electrospray ionisation and collision-induced dissociation tandem mass spectrometry. Rapid Commun. Mass Spectrom. 2001, 15, 1525-1532. [CrossRef]

16. Hvattum, E.; Ekeberg, D. Study of the collision-induced radical cleavage of flavonoid glycosides using negative electrospray ionization tandem quadrupole mass spectrometry. J. Mass Spectrom. 2003, 38, 43-49. [CrossRef]

17. Rice-Evans, C.A.; Miller, N.J.; Paganga, G. Structure-antioxidant activity relationships of flavonoids and phenolic acids. Free Radic. Biol. Med. 1996, 20, 933-956. [CrossRef]

18. Dangles, O.; Fenger, J.-A. The Chemical Reactivity of Anthocyanins and Its Consequences in Food Science and Nutrition. Molecules 2018, 23, 1970. [CrossRef]

19. Turvey, J.R.; Williams, T.P. Sulphates of monsaccharides and derivatives. Part V. Products of sulphation of galactose and glucose. J. Chem. Soc. 1963, 2242-2246. [CrossRef]

20. Needs, P.W.; Kroon, P.A. Convenient syntheses of metabolically important quercetin glucuronides and sulfates. Tetrahedron 2006, 62, 6862-6868. [CrossRef]

21. Mattarei, A.; Biasutto, L.; Romio, M.; Zoratti, M.; Paradisi, C. Synthesis of resveratrol sulfates: Turning a nightmare into a dream. Tetrahedron 2015, 71, 3100-3106. [CrossRef]

22. Iwuchukwu, O.F.; Sharan, S.; Canney, D.J.; Nagar, S. Analytical method development for synthesized conjugated metabolites of trans-resveratrol, and application to pharmacokinetic studies. J. Pharm. Biomed. Anal. 2012, 63, 1-8. [CrossRef]

23. Correia-Da-Silva, M.; Sousa, E.; Pinto, M.M.M. Emerging Sulfated Flavonoids and other Polyphenols as Drugs: Nature as an Inspiration. Med. Res. Rev. 2013, 34, 223-279. [CrossRef]

24. Feliciano, R.P.; Boeres, A.; Massacessi, L.; Istas, G.; Ventura, M.R.; dos Santos, C.N.; Heiss, C.; Rodriguez-Mateos, A. Identification and quantification of novel cranberry-derived plasma and urinary (poly)phenols. Arch. Biochem. Biophys. 2016, 599, 31-41. [CrossRef]

25. Felgines, C.; Talavéra, S.; Gonthier, M.-P.; Texier, O.; Scalbert, A.; Lamaison, J.-L.; Rémésy, C. Strawberry Anthocyanins Are Recovered in Urine as Glucuro- and Sulfoconjugates in Humans. J. Nutr. 2003, 133, 1296-1301. [CrossRef]

26. Kaiser, M.; Müller-Ehl, L.; Passon, M.; Schieber, A. Development and Validation of Methods for the Determination of Anthocyanins in Physiological Fluids via UHPLC-MSn. Molecules 2020, 25, 518. [CrossRef] 